\title{
Type Ia supernovae in a hierarchical galaxy formation model: the Milky Way
}

\author{
Masahiro Nagashima ${ }^{1,2 \star}$ and Takashi Okamoto ${ }^{1} \dagger$ \\ ${ }^{1}$ Department of Physics, University of Durham, South Road, Durham DH1 3LE, United Kingdom \\ ${ }^{2}$ Department of Physics, Graduate School of Science, Kyoto University, Sakyo-ku, Kyoto 606-8502, Japan
}

19 February 2019

\begin{abstract}
We investigate chemical evolution in Milky Way-like galaxies based on the cold dark matter model in which cosmic structures form via hierarchical merging. We introduce chemical enrichment due to type Ia supernovae (SNe Ia) into the Mitaka semi-analytic galaxy formation model developed by Nagashima \& Yoshii. For the first time we treat chemical enrichment due to SNe Ia in a hierarchical galaxy formation model consistently. As a first attempt, we assume all SNe Ia to have the same lifetime, and assume instantaneous recycling for type II supernovae (SNe II). We find that our model reproduce well the metal abundance ratio $[\mathrm{O} / \mathrm{Fe}]$ against $[\mathrm{Fe} / \mathrm{H}]$ and the frequency distribution of iron in the solar neighbourhood. This means that the so-called G-dwarf problem is fully resolved by the hierarchical formation of galaxies. While observations of bulge stars have still uncertainties even for iron, and there are few data for oxygen, our prediction for bulge stars also well agree with those observations. More data in number and accuracy will provide independent and important constraints on galaxy formation. Furthermore, to understand better the chemical enrichment due to SNe Ia in hierarchical galaxy formation, we discuss how physical processes affect the metal abundance ratio by varying the lifetime of $\mathrm{SNe}$ Ia, the star formation timescale and the strength of supernova feedback.
\end{abstract}

Key words: stars: abundances - Galaxy: bulge - Galaxy: disc - Galaxy: evolution - Galaxy: formation - large-scale structure of the universe

\section{INTRODUCTION}

The chemical compositions of stars and gas, as is widely known, provide important clues to understanding the formation and evolution of galaxies. In Particular the abundance ratio of $\alpha$-elements such as oxygen to iron is a useful probe of the formation history of galaxies. The main sources of metals are considered to be supernovae ( $\mathrm{SNe}$ ), especially type II SNe (SNe II) and type Ia SNe (SNe Ia). They have different abundance patterns, the former produces $\alpha$-elements as well as iron and the latter produces hardly any $\alpha$ elements. Therefore, the measurement of $[\alpha / \mathrm{Fe}]$ is closely related to the ratio of SNe II to SNe Ia. While the progenitors of SNe II are massive stars larger than $\sim 10 M_{\odot}$, those of SNe Ia are considered to be white dwarfs in binary systems. Therefore the explosion rate of SNe II is almost proportional to the star formation rate of their progenitor stars at that time, but there is a time lag between $\mathrm{SNe}$ Ia explosions and the formation of their progenitor stars. Since the evolution timescales of those elements are independent and different, we can obtain useful information on galaxy formation and star formation histories from the abundance ratio of those elements.

\footnotetext{
* E-mail: masa@scphys.kyoto-u.ac.jp
}

† E-mail: takashi.okamoto@durham.ac.uk
Because SNe II explode almost instantaneously, the chemical evolution process has usually been formulated by assuming that massive stars immediately explode right after their formation (the instantaneous recycling [IR] approximation)(Tinslev 1980; Lacev \& Fall 1983, 1985). In those works, galaxies are usually assumed to grow up in monolithic clouds, in which timescales of gas infall and star formation (SF) are typically treated as free parameters. Since it is possible to observe and resolve individual stars in the solar-neighbourhood, many studies have focused on evolution of the galactic disc. By adjusting those parameters and by incorporating stellar population synthesis techniques (Arimoto \& Yoshii 1986, 1987), detailed galaxy evolution models that reproduce observations have been developed (Arimoto, Yoshii \& Takahara 1992).

In the framework of such traditional galaxy evolution models, several authors explicitly considered chemical enrichment due to SNe Ia Matteucci \& Greggio 1986; Matteucci \& Francois 1989; Tsuiimoto et al. 1995; Pagel \& Tautvaišiene 1995; Yoshii. Tsuiimoto \& Nomoto 1996). They included delayed production of metals due to $\mathrm{SNe}$ Ia in the galaxy evolution models. By comparing their models with solar neighbourhood stars in frequency distribution of metallicity and abundance ratio such as $[\mathrm{O} / \mathrm{Fe}]$ against metallicity, Pagel \& Tautvaišiene (1995) and 
Yoshii. Tsuiimoto \& Nomoto (1996) inferred a typical lifetime of SNe Ia of $1.3 \sim 1.5$ Gyr.

Considered from the viewpoint of cosmological structure formation, however, these models are still only phenomenological. Recent theoretical and observational studies of structure formation have revealed that the Universe is dominated in mass by cold dark matter (CDM) and that baryonic objects such as galaxies form in virialised objects of dark matter called dark haloes. In the CDM model, since smaller-scale density fluctuations in the early universe have larger fluctuation amplitudes, larger haloes form through mergers of smaller dark haloes (the hierarchical clustering scenario). Since it is difficult for very large galactic gas clouds to form at high redshift because such clouds form by mergers of subgalactic clumps, we need to modify our picture of chemical evolution in galaxies as well as that of galaxies themselves.

Recently, based on a semi-analytic (SA) approach, galaxy formation models in the hierarchical clustering scenario have been developed, in which the formation histories of individual dark haloes are followed by using a Monte Carlo method and physical processes such as gas cooling and star formation are taken into account in the histories of dark haloes. Many authors have found that such SA models reproduce well various characteristics of galaxies at the present and at high redshift such as luminosity functions, gas fractions, size distributions, and faint galaxy number counts (e.g., Kauffmann. White \& Guiderdoni 1993; Cole et al. 1994, 2000; Nagashima. Gouda \& Sugiura 1999; Nagashima et al. 2001, 2002; Nagashima \& Yoshii 2004; Somerville \& Primack 1999; Somerville. Primack \& Faber 2001). In recent SA models, chemical enrichment is considered, but generally only in the framework of the IR approximation. Kauffmann \& Charlot (1998) and Nagashima \& Gouda (2001) investigate colour-magnitude and metallicity-magnitude relations of cluster elliptical galaxies. This is extended to dwarf spheroidals, $M_{B} \sim-10$, by Nagashima \& Yoshii (2004). Kauffmann (1996), Somerville, Primack \& Faber (2001) and Okoshi et al. (2004) consider chemical evolution in spiral galaxies, a part of which should be identified as damped Ly- $\alpha$ systems. Some of them found good agreement with observations.

Pioneering work taking into account chemical enrichment due to SNe Ia in a SA model was carried out by Thomas (1999) and Thomas \& Kauffmann (1999). In those papers, they picked out averaged and individual formation histories of dark haloes, and then, assuming the closed-box chemical evolution, they followed star formation and chemical enrichment histories. Thus, while they took into account merging histories of galaxies, the outflow of gas caused by $\mathrm{SNe}$, or the supernova (SN) feedback, that has been realised to be an important process in galaxy formation, was not considered. As shown by Kauffmann \& Charlot (1998) and Nagashima \& Gouda (2001), SN feedback significantly affects chemical enrichment, at least due to SNe II. This suggests that the closed-box model has limitations in analysing chemical evolution in a realistic situation.

As a complementary approach to SA modelling, chemodynamical simulations including $\mathrm{SNe}$ Ia have been developed (e.g., Raiteri. Villata \& Navarro 1996; Berczik 1999; Kawata 2001; Lia. Portinari \& Carraro 2002; Nakasato \& Nomoto 2003; Kobavashi 2003). Although this approach has an advantage in resolving spatial structure, because of the limitation of numerical resolution due to limited ability of computers, further improvements are still required (Okamoto et al. 2003). On the other hand, the SA model do not have such problems. This is a great advantage for understanding global properties of galaxy formation because in prin- ciple the SA model can investigate from dwarf galaxies to galaxy clusters simultaneously.

In this paper, we construct a fully self-consistent treatment of chemical enrichment with a SA model. The basic model is the Mitaka model preseted by Nagashima \& Yoshii (2004), which includes a Monte Carlo realisation of the merging histories of dark haloes, radiative gas cooling, quiescent star formation and starbursts, mergers of galaxies, chemical enrichment assuming the IR approximation, size estimation of galaxies taking into account the dynamical response to gas removal during starbursts and stellar population synthesis. As a first attempt, we assume that all $\mathrm{SNe}$ Ia have the same lifetime. This is, of course, a rather simplified model. In reality the lifetimes of SNe Ia are considered to have a broad distribution, and there is even a claim that low-metallicity environments inhibit SNe Ia (Kobavashi et al. 1998). Our model, however, has an essential characteristic of SNe Ia, that is, the time lag between star formation and the explosion. It enables us to see how such a delayed explosion of SNe Ia affects the chemical enrichment and abundance pattern in galaxies. Using this model, we focus on the chemical enrichment in the Milky Way (MW). Further comparison to other objects will be done in subsequent papers.

This paper is outlined as follows. In $\S 2$ we describe our SA model. In $\S 3$ we provide a detailed prescription of the chemical enrichment due to SNe Ia. In $\S 4$ we show the luminosity function of galaxies that should be compared with that in the Local Group, and properties of MW-like galaxies. In $\S 5$ we compare our model with observations in the $[\mathrm{O} / \mathrm{Fe}]-[\mathrm{Fe} / \mathrm{H}]$ plane and the $[\mathrm{Fe} / \mathrm{H}]$ distribution. In $\S 6$ we investigate parameter dependences of the main results. In $\S 7$ we provide a summary and conclusions.

\section{SEMI-ANALYTIC MODEL}

Here we briefly describe how to form galaxies in our model, which is based on the Mitaka model (Nagashima \& Yoshii 2004). In a CDM universe, dark matter haloes cluster gravitationally and grow in mass through their mergers, depending on the adopted power spectrum of the initial density fluctuations. In each of the merged dark haloes, radiative gas cooling, star formation, and gas reheating by supernovae occur. The cooled dense gas and stars constitute galaxies. These galaxies sometimes merge together in a common dark halo, and then more massive galaxies form. During these processes, chemical enrichment due to $\mathrm{SNe}$ II is solved by assuming the IR approximation, and the enrichment due to $\mathrm{SNe}$ Ia by using the past star formation histories of individual galaxies (see the next section). Repeating these processes, galaxies form and evolve to the present epoch. The Mitaka model reproduces many observations such as luminosity functions, cold gas fraction, sizes of discs and spheroidals, surface brightnesses, the Faber-Jackson relation, mass-to-light ratio and faint galaxy number counts. The details, apart from chemical enrichment due to SNe Ia are found in Nagashima \& Yoshii (2004).

Throughout this paper, we consider only a $\Lambda$-dominated CDM $(\Lambda \mathrm{CDM})$ cosmology with $\Omega_{0}=0.3, \Omega_{\Lambda}=0.7, h=0.7, \sigma_{8}=$ 0.9 and $\Omega_{\mathrm{b}}=0.02 h^{-2}$, and a power spectrum given by Sugivama (1995), which takes into account the effects of baryon.

The merging histories of dark haloes are calculated by using a method given by Somerville \& Kolatt (1999). Only dark haloes with circular velocity larger than $V_{\text {low }}=40 \mathrm{~km} \mathrm{~s}^{-1}$ are regarded as isolated haloes. While the value of $V_{\text {low }}$ hardly affects the chemical evolution in MW-like galaxies that we consider in this paper, we will compare the luminosity function of galaxies in the Local 
Group (LG) with the model luminosity functions with various $V_{\text {low }}$ in Section 4 The circular velocities of root haloes, which are haloes at $z=0$, is set to be $V_{\text {circ }}=220 \mathrm{~km} \mathrm{~s}^{-1}$, which is nearly equal to the rotation speed of the MW.

Diffuse baryonic gas is shock-heated to the virial temperature estimated from the depth of the gravitational potential well of its host dark halo when it collapses. This is called hot gas, whose mass is $M_{\text {hot }}$. The hot gas dissipates its energy and cools by radiation only for haloes with $V_{\text {circ }} \leqslant V_{\text {cut }}=250 \mathrm{~km} \mathrm{~s}^{-1}$, to avoid formation of unphysically giant galaxies. The cooling rate is estimated by using the metallicity-dependent cooling functions given by Sutherland \& Dopita (1993). This cooled gas concentrates in the vicinity of the centre of the dark halo and constitutes a galactic disc. The mass of cold gas is $M_{\text {cold }}$. Disc stars form by consuming the cold gas with a star formation timescale $\tau_{*}$. In proportion to the star formation rate $\psi=M_{\text {cold }} / \tau_{*}$, massive stars explode as $\mathrm{SNe}$ II. The energy released by SNe II reheats the cold gas and a part of the cold gas is absorbed into the hot gas (SN feedback). The reheating rate is given by $\dot{M}_{\text {reheat }}=\beta \psi$, where $\beta=\left(V_{\text {rot }} / V_{\text {hot }}\right)^{-\alpha_{\text {hot }}}$ and $V_{\text {hot }}$ and $\alpha_{\text {hot }}$ are free parameters. In this paper the star formation timescale is assumed to be $\tau_{*}^{0}(1+\beta)$, where $\tau_{*}^{0}$ is a free parameter, which is called the constant star formation model (CSF) in Nagashima \& Yoshii (2004).

When a dark halo collapses by merging two or more dark haloes, the hot gas components in the progenitor haloes are immediately merged and constitute a hot gas component in the new dark halo. In contrast, galaxies do not merge together immediately. At first we define a central galaxy in the newly collapsing dark halo as the central galaxy in the most massive progenitor halo, and the rest of the galaxies are regarded as satellite galaxies. Two modes of galaxy mergers are considered. One is a merger between a central and satellite galaxies, in which a satellite falls into a central galaxy after losing its energy by dynamical friction (Binnev \& Tremaine 1987). Another is that between satellite galaxies by random collisions (Makino \& Hut 1997). We estimate the timescales of the dynamical friction and the random collisions for all satellites. Satellites with sufficiently short merger timescales compared with the timescale of subsequent collapse of the dark halo merge with a central or other satellite galaxies. If the mass ratio of stars and cold gas of a smaller galaxy to a larger one, $f=m_{\text {small }} / m_{\text {large }}$, is larger than $f_{\text {bulge, }}$ a starburst occurs with $\mathrm{SN}$ feedback and all stars go into the bulge component. Then no cold gas remains. Otherwise the smaller satellite is simply absorbed into the disc component of the central or larger satellite without any activities of star formation. We adopt $f_{\text {bulge }}=0.5$.

In each timestep, the disc size is estimated as a rotationally supported disc under the assumption of specific angular momentum conservation for cooling hot gas. Initially, hot gas is assumed to have the same specific angular momentum as that of its host dark halo. The angular momentum of dark haloes has a log-normal distribution in terms of the so-called dimensionless spin parameter. If the estimated disc size is larger than before, we renew the size. At the same time, we also set the disc rotation velocity to be the same as $V_{\text {circ }}$ of the dark halo. When a major merger occurs, the bulge size is estimated by assuming energy conservation. Then a starburst occurs. Because the mass of the galaxy after the starburst is different from that before, we consider the dynamical response to the mass loss of the size and velocity dispersion. To do this, we use the formalism of the dynamical response taking into account the underlying dark matter potential developed by Nagashima \& Yoshii (2003). This process is required to reproduce observed character- istics of dwarf spheroidals such as surface brightness and velocity dispersion.

Through the above processes, we obtain star formation histories of individual galaxies. From those we estimate the luminosities of galaxies using a stellar population synthesis technique. We adopt the simple stellar populations given by Kodama \& Arimoto (1997).

These procedures are the same as those in Nagashima \& Yoshii (2004). Readers who are interested in the details will find them in that paper. The key parameters of the model are tabulated in Table 1

\section{CHEMICAL ENRICHMENT}

The chemical enrichment is treated in a similar manner to Yoshii. Tsuiimoto \& Nomoto (1996), in which they considered chemical enrichment of oxygen $(\mathrm{O})$ and iron $(\mathrm{Fe})$ due to both $\mathrm{SNe}$ II and SNe Ia using the infall model in the framework of monolithic collapse (Arimoto, Yoshii \& Takahara 1992). When we neglect the mass accretion and assume a fixed lifetime of SNe Ia, $t_{\mathrm{Ia}}$, their basic equations describing the gas fraction, $f_{g}$, and metallicities for the $i$-th elements, $Z_{i}$, are written as

$$
\begin{aligned}
\frac{d f_{g}}{d t} & =-\alpha \psi(t) \\
\frac{d\left(Z_{i} f_{g}\right)}{d t} & =-\alpha Z_{i}(t) \psi(t)+\alpha y_{\mathrm{II}, i} \psi(t)+\alpha y_{\mathrm{Ia}, i} \psi\left(t-t_{\mathrm{Ia}}\right)(2)
\end{aligned}
$$

where $\alpha$ is the locked-up mass fraction in low-mass stars and dead stellar remnants and $y_{\mathrm{II}, i}$ and $y_{\mathrm{Ia}, i}$ are the chemical yields of the $i$ th element produced by SNe II and SNe Ia, respectively (see equations 1 and 2 in their paper). In our model, because of the SN feedback, these equations are modified. During disc star formation or a starburst in a timestep, chemical enrichment due to SNe II is solved by assuming the IR approximation,

$$
\begin{aligned}
\frac{d M_{\mathrm{cold}}}{d t} & =-(\alpha+\beta) \psi(t), \\
\frac{d M_{\mathrm{hot}}}{d t} & =\beta \psi(t), \\
\frac{d\left(M_{\mathrm{cold}} Z_{\mathrm{cold}, i}\right)}{d t} & =-(\alpha+\beta) Z_{\mathrm{cold}, i} \psi(t)+\alpha y_{\mathrm{II}, i} \psi, \\
\frac{d\left(M_{\mathrm{hot}} Z_{\mathrm{hot}, i}\right)}{d t} & =\beta Z_{\mathrm{cold}, i} \psi,
\end{aligned}
$$

where $Z_{\mathrm{cold}, i}$ and $Z_{\mathrm{hot}, i}$ are metallicities for $i$-th element of cold and hot gas, respectively. In addition to this process, metals released from $\mathrm{SNe}$ Ia are put into the cold gas. The mass of $i$-th element released during a timestep $\left[t_{j-1}, t_{j}\right]$ is

$\Delta M_{i}=\alpha y_{\mathrm{Ia}, i} \int_{t_{j-1}}^{t_{j}} \psi\left(t-t_{\mathrm{Ia}}\right) d t$.

In order to estimate this quantity, star formation histories of individual galaxies are stored.

Since one of the main purposes of this study is to compare our results with a monolithic cloud model given by Yoshii. Tsuiimoto \& Nomoto (1996), we adopt the same chemical yields empirically determined by their analysis, which are also tabulated in Table 1 Thus we also impose two nucleosynthesis constraints they adopted: $y_{\mathrm{II}, \mathrm{O}} / y_{\mathrm{II}, \mathrm{Fe}}=$ const., and $y_{\mathrm{Ia}, \mathrm{O}}=0$. We neglect returned mass from $\mathrm{SNe}$ Ia and energy feedback due to $\mathrm{SNe}$ Ia. For simplicity we do not take into account the following effects that might affect chemical evolution of galaxies. One is inhomogeneous star formation in a gas cloud considering propagation of 
Table 1. Model parameters

\begin{tabular}{cccccc}
\hline$V_{\text {hot }}$ & $\alpha_{\text {hot }}$ & $\tau_{*}^{0}$ & $y_{\mathrm{II}, \mathrm{O}}$ & $y_{\mathrm{II}, \mathrm{Fe}}$ & $y_{\mathrm{Ia}, \mathrm{Fe}}$ \\
\hline $150 \mathrm{~km} \mathrm{~s}^{-1}$ & 4 & $1.5 \mathrm{Gyr}$ & $7.19 \times 10^{-3}$ & $3.40 \times 10^{-4}$ & $6.67 \times 10^{-4}$ \\
\hline
\end{tabular}

star formation sites, which is another way to extend the simple traditional galaxy evolution model (Tsuiimoto, Shigevama \& Yoshii 1999; Ishimaru \& Wanaio 1999). Although it will probably provide a broader distribution in metal abundances, we concentrate, in this paper, on how the hierarchical formation process affects the chemical enrichment. Another is the metallicity effect that a low metallicity environment inhibits the explosion of SNe Ia Kobavashi et al. 1998), which might affect the estimation of $t_{\text {Ia }}$. These effects would be worth considering in future.

\section{LUMINOSITY FUNCTION AND GLOBAL CHARACTERISTICS OF MW-LIKE GALAXIES}

The model we use here is the same as that in Nagashima \& Yoshii (2004) except for the chemical enrichment process, which do not affect any results in that paper. Therefore we do not repeat showing the same figures as shown in that paper.

In the following, we pick out galaxies in dark haloes of $V_{\text {circ }}=$ $220 \mathrm{~km} \mathrm{~s}^{-1}$ whose central galaxies are identified as spiral galaxies with $B$-band bulge-to-total luminosity ratio smaller than 0.4 (Baugh, Cole \& Frenk 1996) and with $-22.1 \leqslant M_{I}-5 \log h \leqslant$ -21.6 (Somerville \& Primack 1999). Hereafter, we call a dark halo satisfying the above criteria a LG-halo. We realise 100 LG-haloes and average over the realisation.

Before entering the details, we see the characteristics of MWlike galaxies and their satellites. In Figure 1 we show a luminosity function of galaxies in LG-halos. The solid line indicates the model luminosity function and the histogram the observed one given by van den Bergh (1992). Our model shows a broad agreement with the observed luminosity function in the LG at $M_{B} \lesssim-15$, although at fainter magnitude too many galaxies are predicted. Although the observed luminosity functions actually depend on the definition of the Local Group, our conclusion about the chemical evolution of the MW-like galaxies does not change as shown below. The detailed discussion is given by Benson et al. (2002b).

Such overabundance is easily eliminated by taking into account some physical or observational effects as shown by many authors. One of the important effects is photoionisation due to ultraviolet (UV) background or reionisation (e.g., Nagashima, Gouda \& Sugiura 1999; Nagashima \& Gouda 2001; Somerville 2002; Benson et al. 2002a b, 2003). Here we show the effect of the Jeans mass increasing due to reionisation in a simple way by changing $V_{\text {low }}$. Note that in reality this effect should turn on only after the epoch of reionisaion. Indeed, this simple model is similar to Kauffmann. White \& Guiderdoni (1993), in which they assumed that gas cooling is prohibited in dark haloes with $V_{\text {circ }} \leqslant 150 \mathrm{~km} \mathrm{~s}^{-1}$ at redshifts between 1.5 and 5 to be consistent with the LG luminosity function in the standard CDM model. In Figure 1b, we show luminosity functions with different $V_{\text {low }}$. As shown in the panel the solid, dashed, dot-dashed and dotted lines indicate $V_{\text {low }}=40$ (the reference model), 30, 50 and 70 $\mathrm{km} \mathrm{s}^{-1}$, respectively. Evidently large $V_{\text {low }}$ inhibits the formation of dwarf galaxies. Another effect is incompleteness in the observations. Nagashima \& Yoshii (2004) predict that there are many

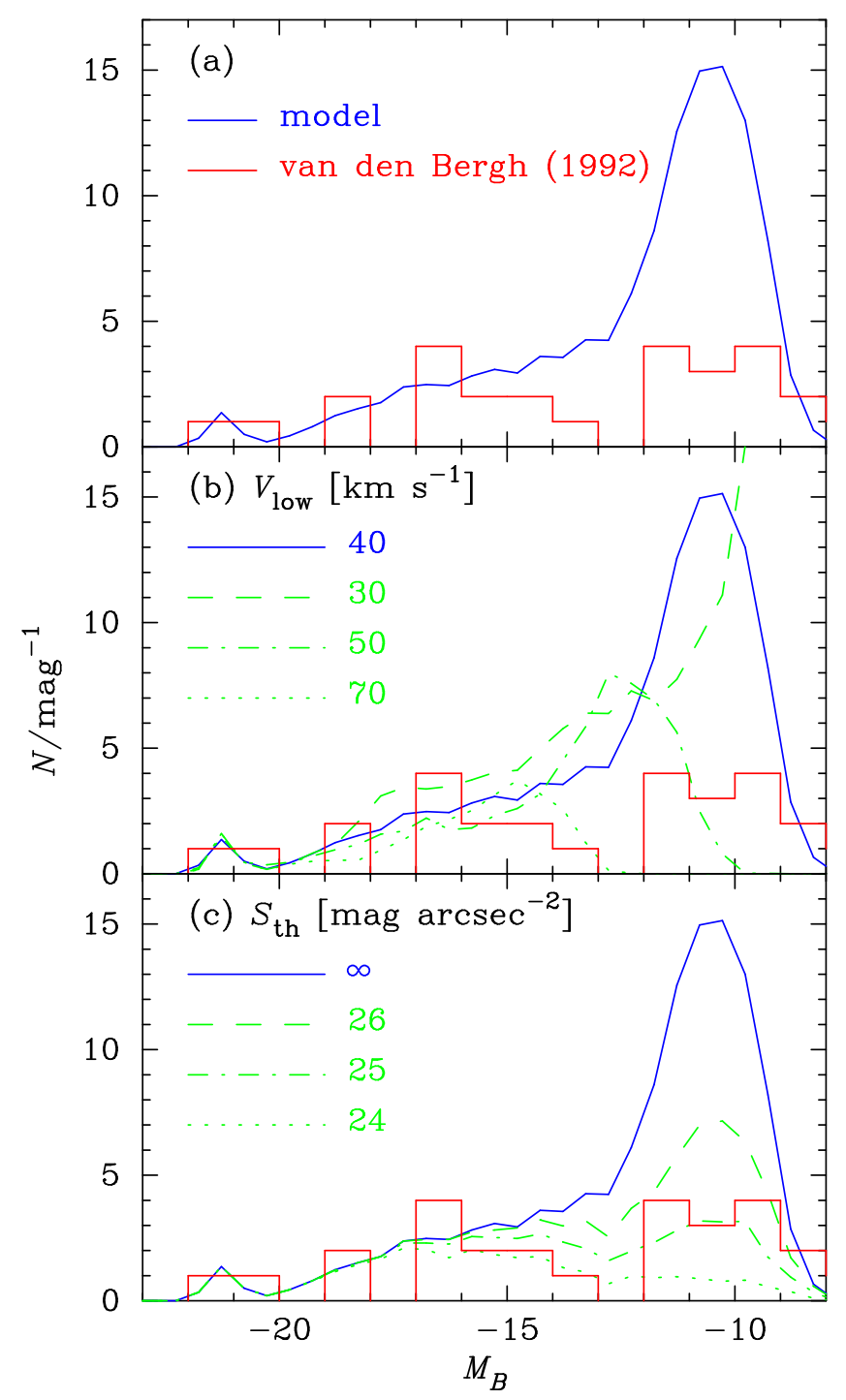

Figure 1. Luminosity function in the LG. (a) The solid line indicates the reference model. The histogram denotes observed luminosity function in the LG given by van den Bergh (1992). (b) Dependence on $V_{\text {low }}$. The solid line is the same as model in (a), the reference model of $V_{\text {low }}=40 \mathrm{~km} \mathrm{~s}^{-1}$. The dashed, dot-dashed and dotted lines indicate variants of the reference model with $V_{\text {low }}=30,50$ and $70 \mathrm{~km} \mathrm{~s}^{-1}$. (c) Dependence on $S_{\mathrm{th}}$. The solid line is the same as model in (a), the reference model of $S_{\mathrm{th}}=\infty$. The dashed, dot-dashed and dotted lines indicate variants of the reference model with $S_{\mathrm{th}}=26,25$ and $24 \mathrm{mag}_{\operatorname{arcsec}}{ }^{-2}$, respectively.

dwarf galaxies with surface brightnesses too low to detect. In the bottom panel, Figure 11; we show luminosity functions with different threshold surface brightnesses, $S_{\mathrm{th}}$, which determines the lowest surface brightness of galaxies to be detected. The solid, dashed, dot-dashed and dotted lines indicate $S_{\mathrm{th}}=\infty, 26,25$ and 24 mag $\operatorname{arcsec}^{-2}$, respectively, where surface brightness is defined as that within the effective radius. In addition, some of these low sur- 


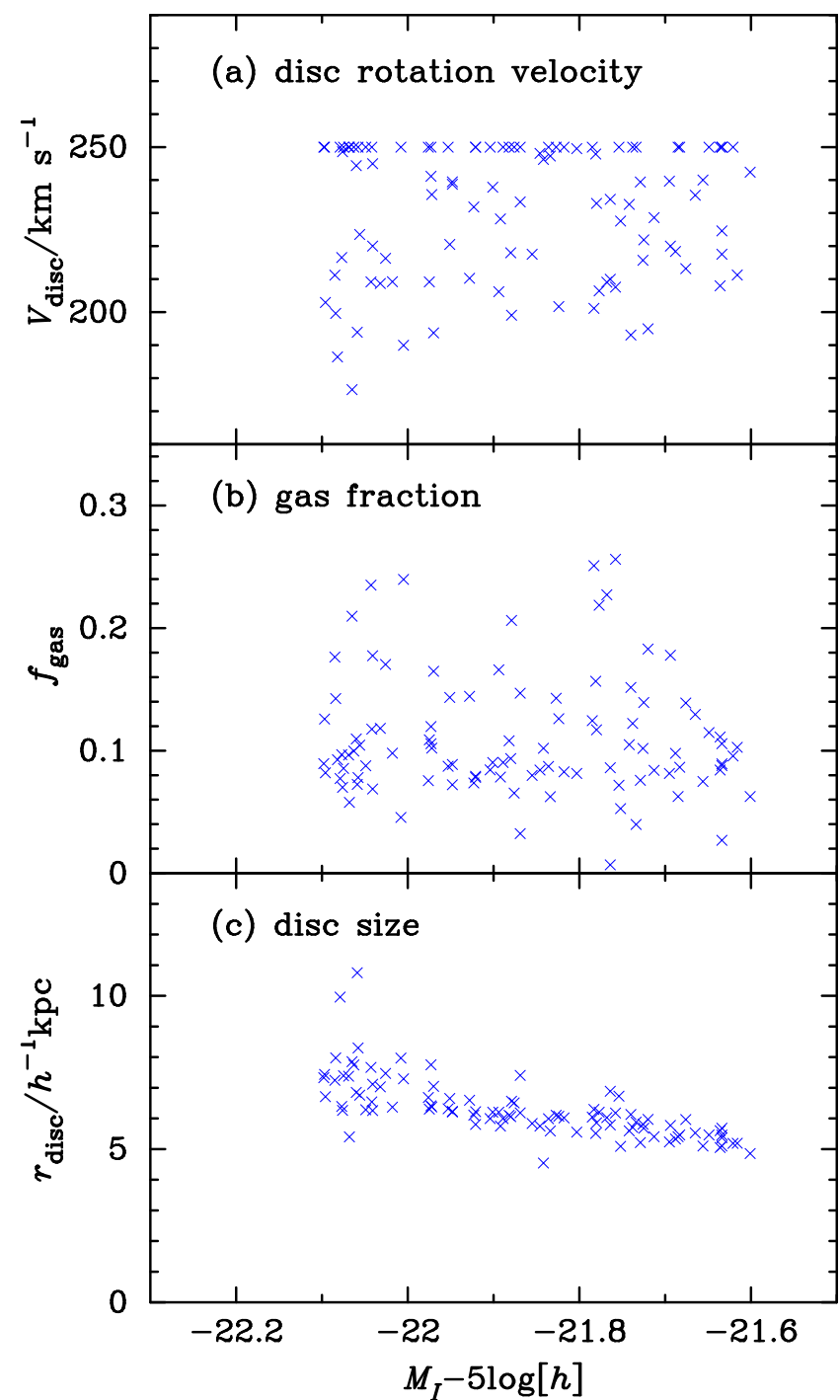

Figure 2. Characteristics of MW-like galaxies. The crosses indicate each MW-like galaxy. (a) Disc rotation velocity $V_{\text {rot. }}$ (b) Cold gas fraction. (c) Effective radius of discs.

face brightness galaxies might be disrupted by tidal interaction with other galaxies (e.g., Martine et al. 2003). These figures suggest that the so-called satellite-galaxy problem might not be so serious, at least for luminous objects. Anyway, changing $V_{\text {low }}$ and $S_{\text {th }}$ hardly affects our results on chemical enrichment of the MW-like galaxies.

In Figure 2 we show the disc rotation velocity, $V_{\text {rot }}$, cold gas fraction, $f_{\text {gas }}$, and effective radius of the disc, $r_{\text {disc }}$, of the MW-like galaxies. The crosses denote individual MW-like galaxies. Since the disc rotation velocity is adjusted to be the circular velocity of the host dark halo only when the mass of disc increases, it is not always the same as $V_{\text {circ }}$, which is chosen to be $220 \mathrm{~km} \mathrm{~s}^{-1}$ at $z=0$. Some of the galaxies have $V_{\text {rot }}=V_{\text {cut }}=250 \mathrm{~km} \mathrm{~s}^{-1}$. This is a kind of artifact due to the cooling cut-off at $V_{\text {cut }}$. The rotation velocity obtained is distributed around the value $220 \mathrm{~km} \mathrm{~s}^{-1}$ that is similar to the observed rotation velocity of the Galactic disc. The cold gas fraction is about $10-15 \%$ in mass, and the effective radius is around $7 h^{-1} \mathrm{kpc}$. These are very similar to the observed properties of the MW. Thus our MW-like galaxies are good probes enough to investigate the chemical enrichment in the MW.

\section{COMPARISON WITH SOLAR NEIGHBOURHOOD AND BULGE STARS}

In this model we treat bulge and disc stars separately, so hereafter we show results for both separately, keeping in mind there are still large uncertainties in observation of bulge stars.

In Figure 3a we show the distribution of disc stars in the $[\mathrm{O} / \mathrm{Fe}]-[\mathrm{Fe} / \mathrm{H}]$ plane by contours. The levels of contours drawn by the thin solid and dashed lines indicate $0.5,0.4,0.3,0.2$ and 0.1 , and 0.02 and 0.005 times the largest number of stars in grids, respectively. The solid lines indicate the $k=1$ model given by Yoshii, Tsuiimoto \& Nomoto (1996) (see Tables 1 and 2 in their paper for details). The crosses and filled triangles are observed data for solar-neighbourhood stars given by Meléndez \& Barbuv (2002) and Edvardsson et al. (1993), respectively. As is clearly shown in this panel, our model reproduce well the observations of disc stars including their dispersion. The apparent discrepancy at $[\mathrm{Fe} / \mathrm{H}] \lesssim-2.5$ is caused by the first nucleosynthesis constraint, $y_{\mathrm{II}, \mathrm{O}} / y_{\mathrm{II}, \mathrm{Fe}}=$ const. for all SNe II (see Section 3). At the break point the model prediction gives slightly higher $[\mathrm{O} / \mathrm{Fe}]$ than observations. Probably if the assumption of a constant $t_{\text {Ia }}$ were relaxed, the abundance ratio at there would decrease as suggested by Yoshii. Tsuiimoto \& Nomoto (1996).

It should be noted that a small radial abundance gradients of metals in the galactic disc are suggested by observations, $\sim$ $-0.085 \mathrm{dex} \mathrm{kpc}^{-1}$ for iron and $\sim-0.07 \mathrm{dex} \mathrm{kpc}^{-1}$ for oxygen (Maciel 2001). This might cause a systematic difference in the metallicity distribution when averaged over the whole disc. In that case, we will be needed to change the values of chemical yields, which are empirically determined by Yoshii. Tsuiimoto \& Nomoto (1996) to produce an agreement with the observations for solarneighborhood stars.

Bulge stars are also distributed like disc stars in the $[\mathrm{O} / \mathrm{Fe}]-$ $[\mathrm{Fe} / \mathrm{H}]$ plane but the range is slightly different as shown in Figure 3 $\mathrm{b}$. In particular, although the fraction is low, there are many oxygen-enhanced stars at $[\mathrm{Fe} / \mathrm{H}] \simeq 0$. Interestingly, Rich \& McWilliam (2000) found that [O/Fe] for bulge stars, shown by filled inverse triangles, has a broadly similar trend with $[\mathrm{Fe} / \mathrm{H}]$ to disc stars, but some of them show oxygen-enhancement, $[\mathrm{O} / \mathrm{Fe}] \simeq$ 0.3 even at $[\mathrm{Fe} / \mathrm{H}] \simeq 0$. Recent high-resolution spectroscopic observations of bulge stars by McWilliam. Rich \& Smecker-Hane (2003) reveal a similar trend of $[\mathrm{Mn} / \mathrm{Fe}]$ to that for disc stars. While they observed less than 10 stars, their observations may support the success of our modelling. More samples will provide important constraints on bulge formation.

In Figures 3, and $d$, we show the frequency distributions of stars against $[\mathrm{Fe} / \mathrm{H}]$ for disc and bulge stars, respectively. The solid lines denote the model prediction. The filled squares and crosses in the panel (c) and the filled squares, crosses and pluses in the panel (d) indicate observed $[\mathrm{Fe} / \mathrm{H}]$ distributions given by Wvse \& Gilmore (1995) and Edvardsson et al. (1993) for disc stars and Zoccali et al. (2003), Ramírez et al. (2000) and Sadler, Rich \& Terndrup (1996) for bulge stars, respectively. All of these distributions are normalised for the integrate over the whole range to be unity. The distribution for disc stars shows an excellent agreement with the observed distributions. Thus, both these agreements in abundance ratio and frequency distribution suggest that our SA model describes well the formation of the galactic disc.

While the predicted distribution of $[\mathrm{Fe} / \mathrm{H}]$ for bulge stars is rather similar to that for disc stars, the width is clearly wider than that of disc stars. The observations of bulge stars show a similar tendency to the model, although there seem to be still uncertainties in 


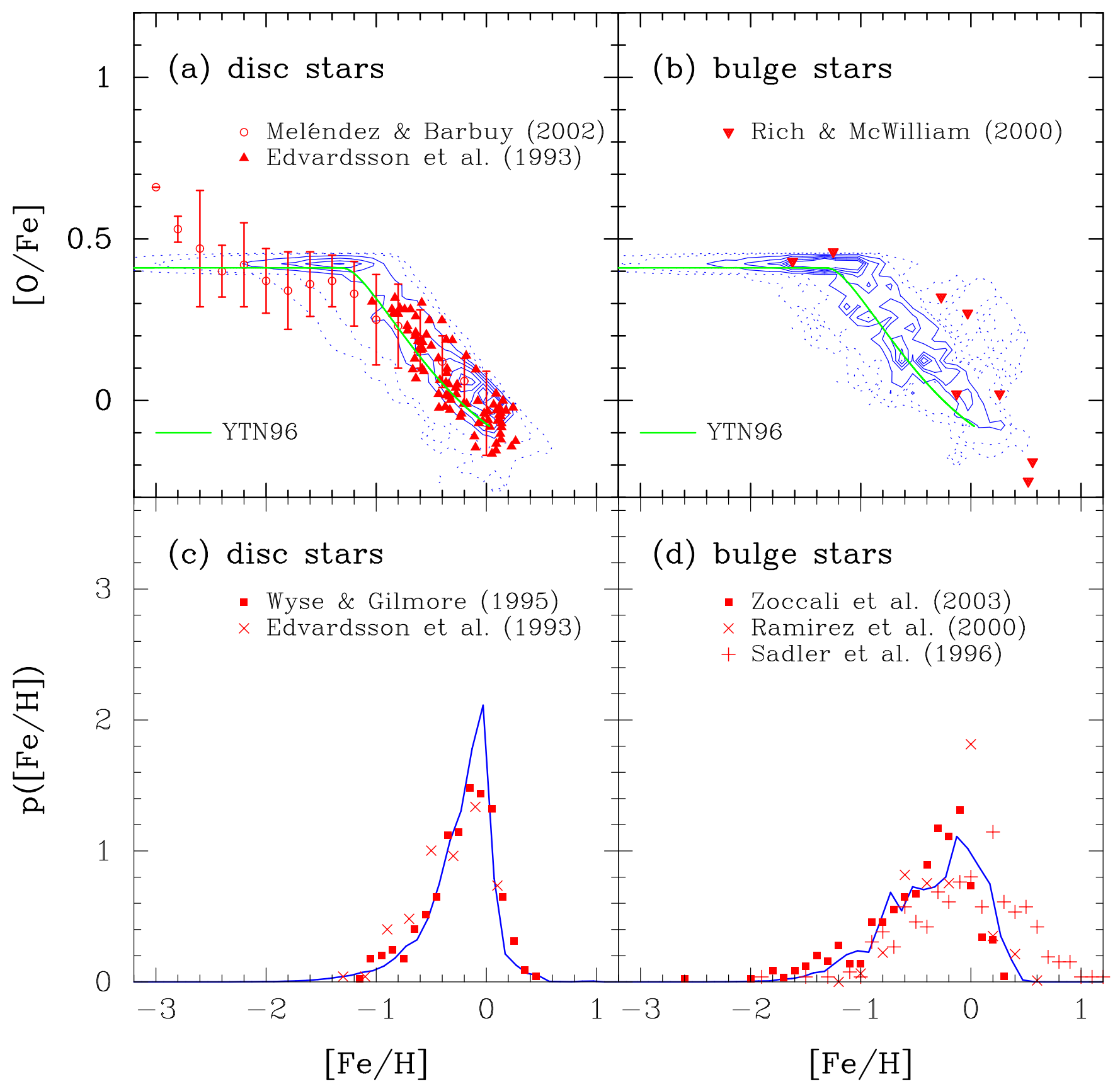

Figure 3. (a) $[\mathrm{O} / \mathrm{Fe}]$ distribution against $[\mathrm{Fe} / \mathrm{H}]$ for disc stars. The levels of contours drawn by the thin solid and dashed lines indicate $0.5,0.4,0.3,0.2$ and 0.1 , and 0.02 and 0.005 times the largest number of stars in grids, respectively. The solid curve indicates a chemical enrichment model based on a monolithic cloud collapse model given by Yoshii. Tsuiimoto \& Nomoto (1996). The circles with errorbars and filled triangles denote observations by Meléndez \& Barbuv (2002) and Edvardsson et al. (1993), respectively. (b) $[\mathrm{O} / \mathrm{Fe}]$ distribution against $[\mathrm{Fe} / \mathrm{H}]$ for bulge stars. The levels of contours and the solid curve are the same as the panel (a). The filled inverse triangles denote observational data by Rich \& McWilliam (2000). (c) Frequency distribution of [Fe/H] for disc stars. The solid line indicates the model prediction for disc stars. The filled squares and crosses denote observations by Wvse \& Gilmore (1995) and Edvardsson et al. 1993), respectively. (d) Frequency distribution of [Fe/H] for bulge stars. The solid line indicates the model prediction for bulge stars. The filled squares, crosses and pluses denote observations by Zoccali et al. (2003), Ramírez et al. (2000) and Sadler. Rich \& Terndrup (1996), respectively.

observations outside the range $-1 \lesssim[\mathrm{Fe} / \mathrm{H}] \lesssim 0$. Keeping in mind differences between observational data, the model prediction for bulge stars also agrees well with the observed data. In another approach, a recent chemo-dynamical simulation of the galactic bulge by Nakasato \& Nomoto (2003) also predicts distribution of stars in $[\mathrm{O} / \mathrm{Fe}]-[\mathrm{Fe} / \mathrm{H}]$ plane and in number against $[\mathrm{Fe} / \mathrm{H}]$. In their results, there are more oxygen-enhanced stars than in the observa- tion by Rich \& McWilliam (2000) and our prediction. At the same time, their derived $[\mathrm{Fe} / \mathrm{H}]$ distribution is similar to ours, although the number of high $[\mathrm{Fe} / \mathrm{H}]$ stars is a little larger. Thus, although more data will be required to constrain the model of bulge formation, we can say that both our SA model and the recent numerical simulation broadly agree with the current observations.

Our results suggest that the formation of spiral galax- 
ies is well approximated by the traditional infall model because our model provides similar results to those given by Yoshii. Tsuiimoto \& Nomoto (1996). This is consistent with the result by Baugh. Cole \& Frenk (1996), in which they traced formation histories of individual galaxies and found similar star formation histories of spiral galaxies to the traditional infall model.

\section{PARAMETER DEPENDENCE}

Here we investigate how the stellar metallicity depends on the fundamental model parameters such as the SN feedback and star formation timescale, as well as the lifetime of SNe Ia.

\subsection{Lifetime of SNe Ia}

Firstly, we vary the lifetime of SNe Ia, $t_{\mathrm{Ia}}$. In Figures 4 and 5 we show the same model as in Figure 3 but for $t_{\mathrm{Ia}}=0.5$ and $3 \mathrm{Gyr}$, respectively. In the case of the shorter $t_{\mathrm{Ia}}$, because enrichment due to $\mathrm{SNe}$ Ia begins earlier, the break point in the $[\mathrm{O} / \mathrm{Fe}]-$ $[\mathrm{Fe} / \mathrm{H}]$ plane moves toward lower $[\mathrm{Fe} / \mathrm{H}]$. On the other hand, in the longer $t_{\mathrm{Ia}}$ case, the iron abundance initially increases only due to $\mathrm{SNe}$ II, so the break point moves toward higher $[\mathrm{Fe} / \mathrm{H}]$. This dependence is basically the same as that shown in analyses using the traditional model. This also suggests the similarity of formation of spiral galaxies between the hierarchical and traditional models.

In the shorter $t_{\text {Ia }}$ case, an interesting feature in abundance ratio emerges there. Bulge stars has a kind of U-shaped distribution with a minimum at $[\mathrm{Fe} / \mathrm{H}] \sim-0.5$ in $[\mathrm{O} / \mathrm{Fe}]-[\mathrm{Fe} / \mathrm{H}]$ plane. In this case, iron enrichment due to $\mathrm{SNe}$ Ia is almost completed at very high redshift, at which time a large amount of cold gas remains in galaxies. Hence most of the stars existing at present form in subsequent starburst due to major mergers between such gas-rich systems. Because starbursts tends to make stars at its late phase with the abundance pattern of SNe II, if the system is gas-rich, many oxygen-enhanced iron-rich stars form during the starburst. Thus this provides a constraint on the lifetime of $\mathrm{SNe} I$ a, that is, $t_{\mathrm{Ia}}$ should be rather long, $\gtrsim 1 \mathrm{Gyr}$.

The dependence of the $[\mathrm{Fe} / \mathrm{H}]$ distribution on $t_{\mathrm{Ia}}$ is not as simple as the abundance ratio. These figures show that the lifetime $t_{\text {Ia }}$ seems to determine the width of $[\mathrm{Fe} / \mathrm{H}]$ distribution, that is, a shorter $t_{\text {Ia }}$ makes the $[\mathrm{Fe} / \mathrm{H}]$ distribution narrower. It could be related to the epoch of star formation. In the shorter $t_{\text {Ia }}$ case, many intermediate stars explode as SNe Ia and begin to release metals into the interstellar medium before the major epoch of star formation compared with the reference model, therefore most of the stars have similar iron abundances, which makes the $[\mathrm{Fe} / \mathrm{H}]$ distribution narrow. In contrast, in the longer $t_{\mathrm{Ia}}$ case, some of the stars form in iron-poor environments before most of the iron is released, and some of the stars form in iron-rich environments because at late epochs the gas fraction is low and iron is released into gas-poor systems. Thus the $[\mathrm{Fe} / \mathrm{H}]$ distribution has a large width.

\subsection{Star formation timescale}

Next, we investigate the dependence on star formation timescale. In Figures 6 and 7 we show models which are the same as the reference model, except for having twice and half the star formation timescale of the reference model, $\tau_{*}^{0}=2.6$ and $0.65 \mathrm{Gyr}$, respectively.

As seen in Figure 6], in the longer star formation timescale case, the break point in $[\mathrm{O} / \mathrm{Fe}]-[\mathrm{Fe} / \mathrm{H}]$ plane for bulge stars moves

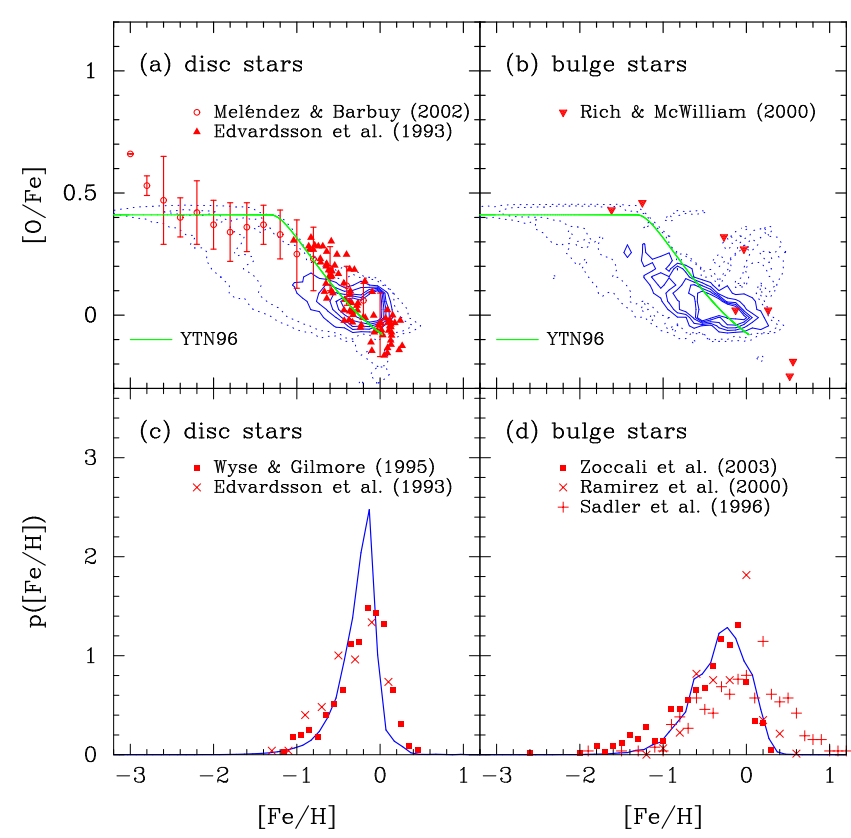

Figure 4. The same as Figure 3 but for $t_{\mathrm{Ia}}=0.5 \mathrm{Gyr}$.

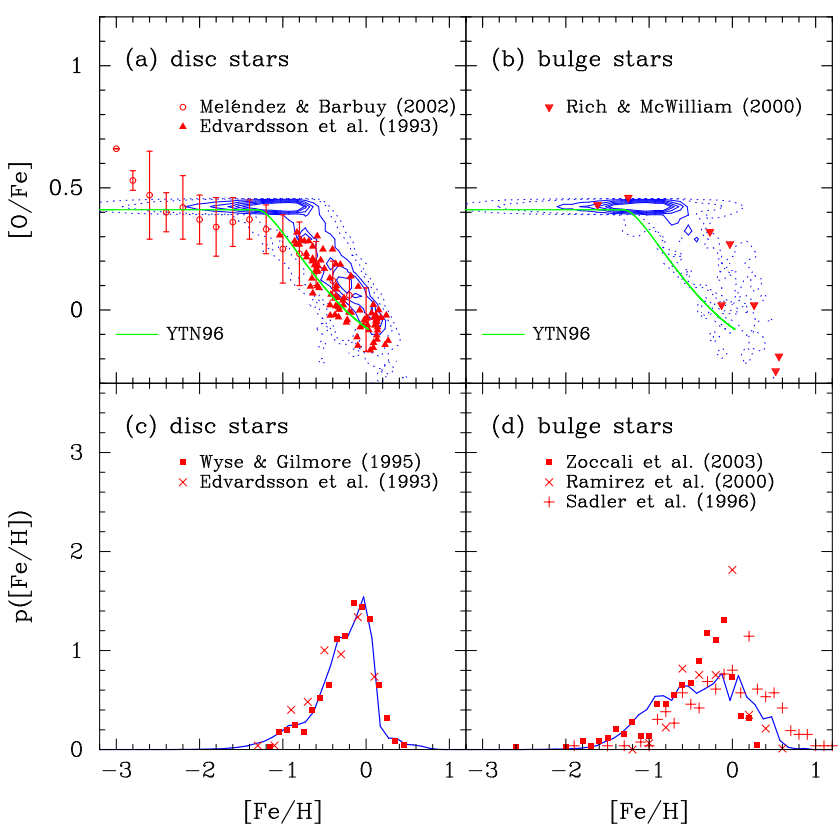

Figure 5. The same as Figure 3 but for $t_{\mathrm{Ia}}=3 \mathrm{Gyr}$.

towards lower $[\mathrm{Fe} / \mathrm{H}]$, because the star formation epoch shifts to lower redshift and then the enrichment due to SNe Ia effectively begins earlier. In the case of a shorter star formation timescale, by contrast, the break point moves towards higher $[\mathrm{Fe} / \mathrm{H}]$. Thus we can say that the position of the break point is generally determined by a combination of the lifetime of $\mathrm{SNe}$ Ia and the star formation timescale, such as $t_{\mathrm{Ia}} / \tau_{*}^{0}$. In the longer $\tau_{*}^{0}$ case, there is a broader tail showing oxygen-enhancement at $[\mathrm{Fe} / \mathrm{H}] \sim 0$ than in the reference model. This is for the same reason as the case of shorter $t_{\text {Ia }}$. The difference from the case with shorter $t_{\mathrm{Ia}}$ is that the distribution is not U-shaped but an inverse triangle, because the SNe Ia continue enrichment at lower redshifts.

The $[\mathrm{Fe} / \mathrm{H}]$ distribution shows a different tendency from the 


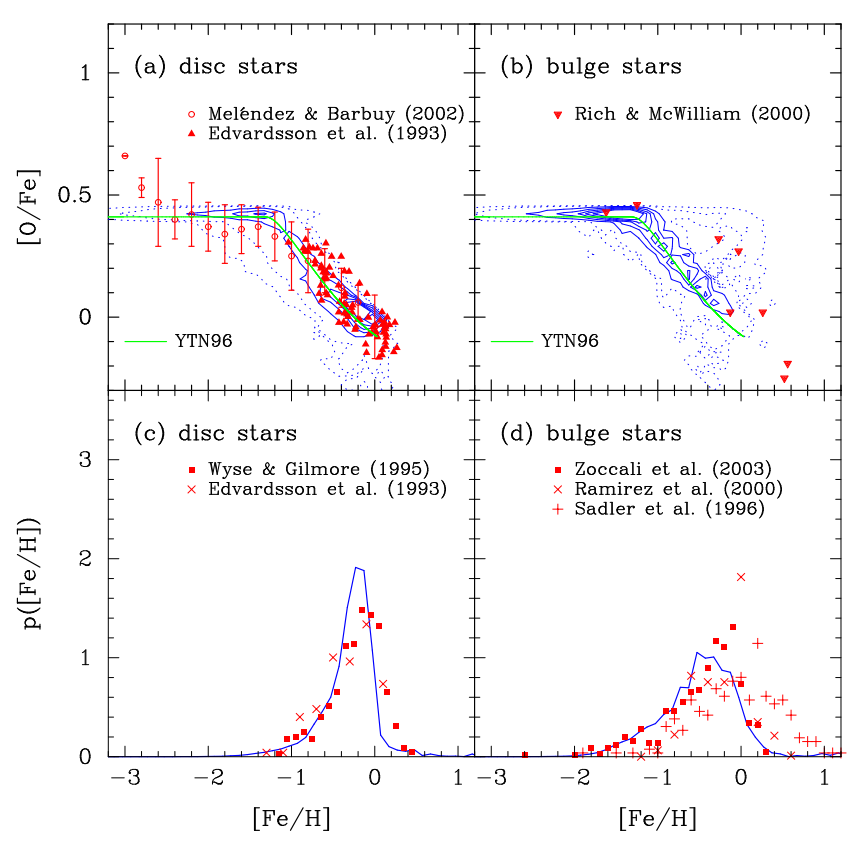

Figure 6. The same as Figure 3 but for $\tau_{*}^{0}=2.6 \mathrm{Gyr}$.

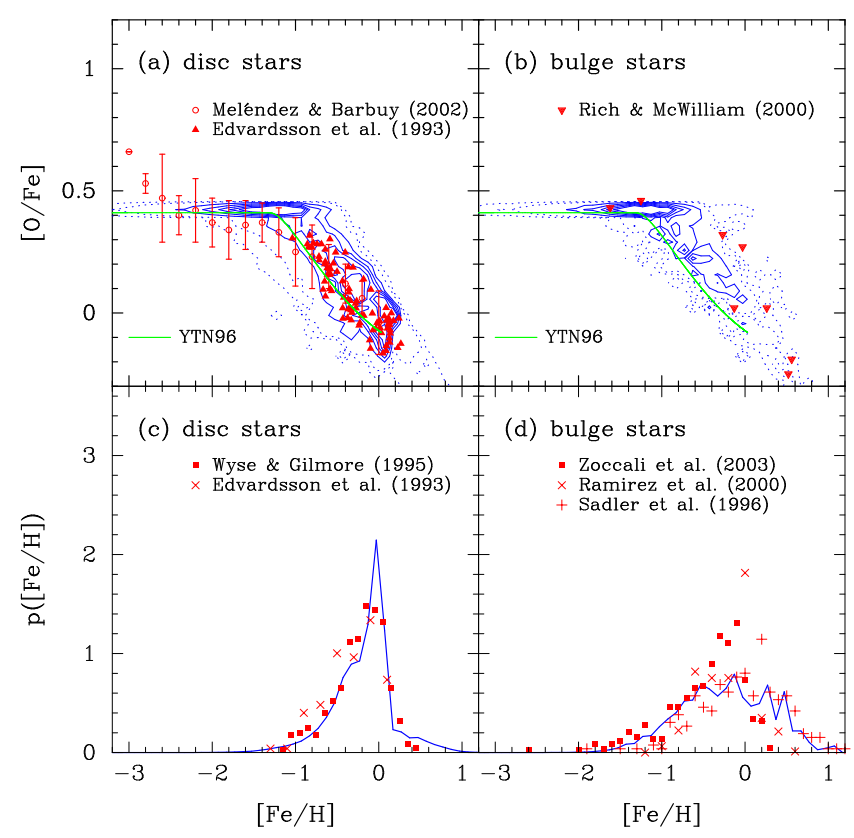

Figure 7. The same as Figure 3 but for $\tau_{*}^{0}=0.65 \mathrm{Gyr}$.

dependence on $t_{\mathrm{Ia}}$. In the case of longer and shorter $\tau_{*}^{0}$, the distributions typically have lower and higher $[\mathrm{Fe} / \mathrm{H}]$, respectively. Generally a longer star formation timescale results in a larger cold gas fraction. Then, with the longer star formation timescale, the metallicity becomes lower than in a model with a shorter star formation timescale. In addition, such a longer star formation timescale decreases the amount of stars, which means that fewer metals are released from $\mathrm{SNe}$ Ia. Thus the star formation timescale has a different effect from the lifetime of SNe Ia.

In the reference model, the star formation timescale is assumed to be constant with redshift. It is interesting to see how the redshift dependence affects the chemical enrichment. In Nagashima \& Yoshii (2004), another formulation for the star for-

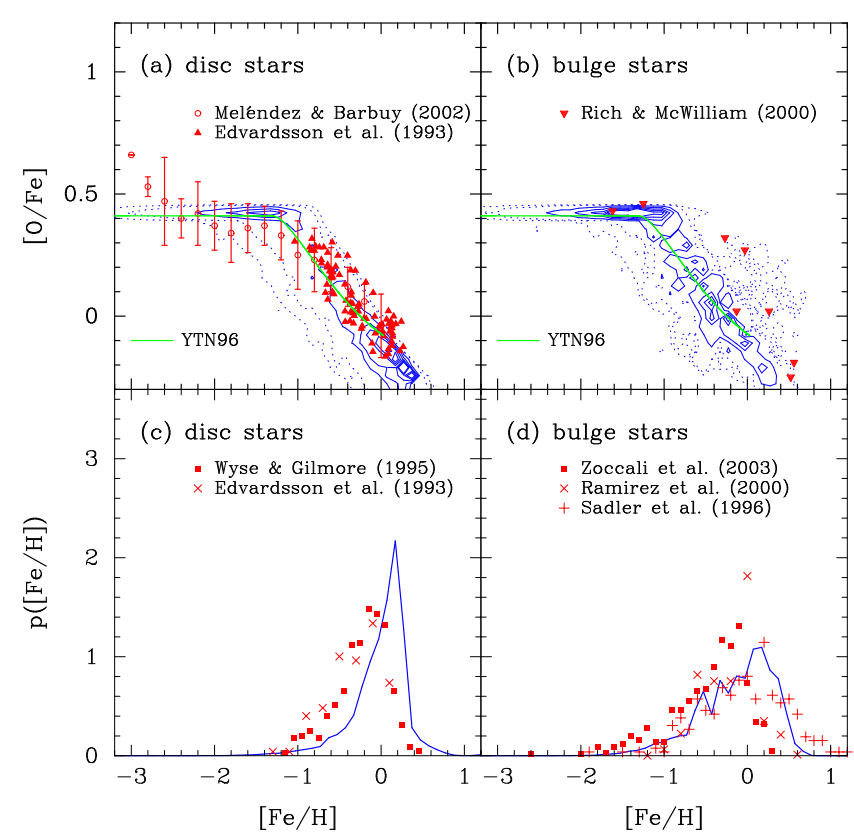

Figure 8. The same as Figure 3 but for the DSF model.

mation timescale is considered; it is proportional to the dynamical timescale of galactic disc, which is called the dynamical star formation (DSF) timescale model in that paper. Because we assume the disc rotation velocity to be almost the same as the circular velocity of dark haloes, which becomes larger toward higher redshift, the dynamical timescale becomes shorter toward higher redshift. Figure 8 shows the results of adopting the DSF model, in which the star formation timescale at present is $\tau_{*}^{0}=1.7 \mathrm{Gyr}$, so as to produce an agreement of cold gas fraction in spiral galaxies with observation. We find that although the position of the break point in $[\mathrm{O} / \mathrm{Fe}]-[\mathrm{Fe} / \mathrm{H}]$ plane is not changed, the slope of $[\mathrm{O} / \mathrm{Fe}]$ against $[\mathrm{Fe} / \mathrm{H}]$ becomes slightly steeper. We also find that many stars have large iron abundance. In the DSF model, since most of stars form at higher redshift than in the reference model due to the shorter star formation timescale at high redshift, enrichment of iron happens quickly. Thus the slope is steeper and the typical $[\mathrm{Fe} / \mathrm{H}]$ is larger than in the reference model.

\subsection{Supernova feedback}

It has been widely realised that $\mathrm{SN}$ feedback significantly affects galaxy properties. Strong energy feedback suppresses chemical enrichment due to SNe II because star formation is suppressed by expelling cold gas, and also most of the metals are expelled into the hot gas (Kauffmann \& Charlot 1998; Nagashima \& Gouda 2001). Thus it is worthwhile to investigate how SN feedback affects the enrichment due to both SNe II and SNe Ia.

In Figures 9 and 10 we show the same figures as Figure 3 but for $V_{\text {hot }}=280$ (strong feedback) and 70 (weak feedback) $\mathrm{km} \mathrm{s}^{-1}$, respectively, where $V_{\text {hot }}$ determines the typical scale at which SN feedback becomes effective. As clearly shown in the bottom panels, the $[\mathrm{Fe} / \mathrm{H}]$ distributions for both disc and bulge stars are strongly affected by $\mathrm{SN}$ feedback. Strong feedback greatly suppresses formation of metal-rich stars and thus the chemical enrichment. In contrast, there are too few metal-poor stars in the case of weak SN feedback compared with the observed distributions, while the difference from the reference model is smaller than that of the strong 


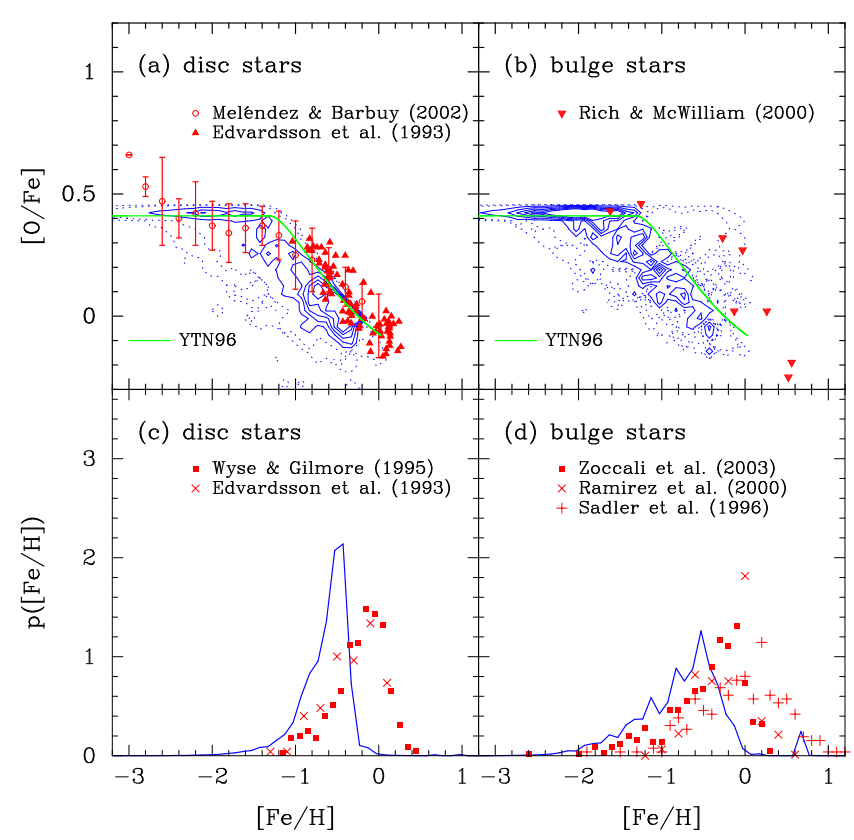

Figure 9. The same as Figure 3 but for $V_{\text {hot }}=280 \mathrm{~km} \mathrm{~s}^{-1}$.

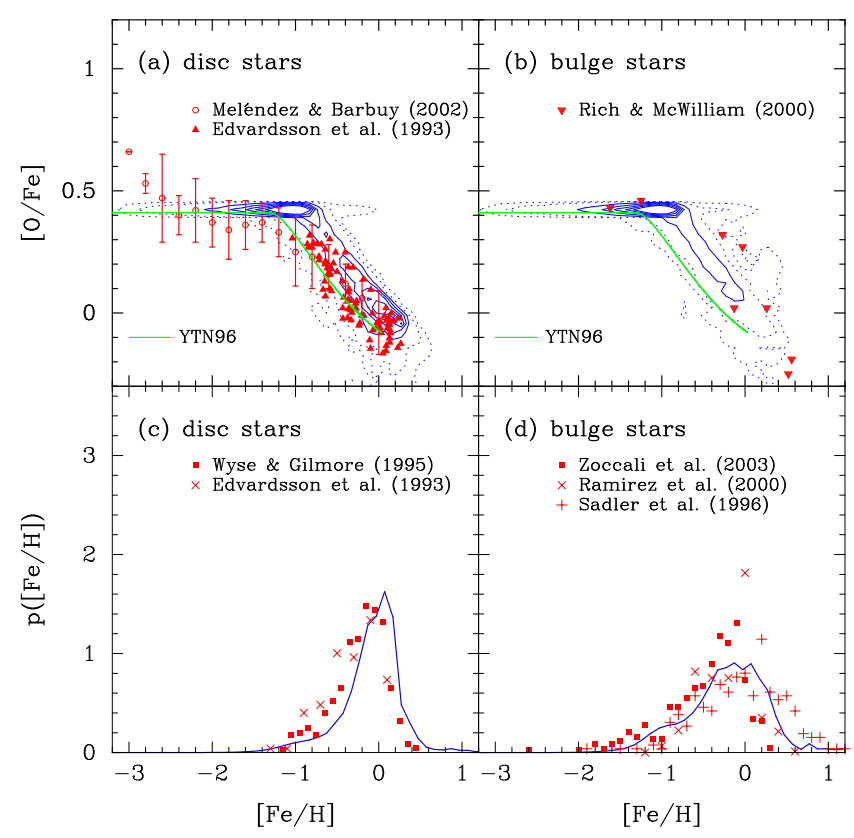

Figure 10. The same as Figure 3 but for $V_{\text {hot }}=70 \mathrm{~km} \mathrm{~s}^{-1}$.

SN feedback case. It should be noted that our MW-like galaxies have $V_{\text {rot }} \simeq 220 \mathrm{~km} \mathrm{~s}^{-1}$ at low redshift. The values of $V_{\text {hot }}$ for both the reference model and the weak SN feedback case are less than the rotation velocity, while in the strong SN feedback case $V_{\text {hot }}$ is larger than the rotation velocity. That means that $\mathrm{SN}$ feedback is very efficient even at low redshift in the strong feedback case. Therefore the difference of the strong SN feedback case from the reference model is larger.

The stronger the $\mathrm{SN}$ feedback is, the lower the $[\mathrm{Fe} / \mathrm{H}]$ at the break point is. Consequently, strong and weak $\mathrm{SN}$ feedback seem to have similar effects in the $[\mathrm{O} / \mathrm{Fe}]-[\mathrm{Fe} / \mathrm{H}]$ plane to those of short and long $t_{\text {Ia }}$, and long and short $\tau_{*}^{0}$. Since strong $\mathrm{SN}$ feedback suppresses the chemical enrichment due to SNe II, the enrichment due to $\mathrm{SNe}$ Ia begins at a lower $[\mathrm{Fe} / \mathrm{H}]$. These apparently correspond to longer and shorter star formation timescales, respectively.

\section{SUMMARY}

We have explored chemical enrichment due to both SNe II and $\mathrm{SNe}$ Ia in Milky Way-like galaxies in the semi-analytic galaxy formation model. Our treatment of SNe Ia is fully consistent with the galaxy formation model, that is, for the first time based on a $\Lambda \mathrm{CDM}$ model we solve for the recycling of each element among stars, cold gas and hot gas. As a first attempt at constructing such a consistent model, we have assumed that all $\mathrm{SNe}$ Ia have the same lifetime, $t_{\mathrm{Ia}}$, that abundance patterns of metals from $\mathrm{SNe} \mathrm{Ia}$ and $\mathrm{SNe}$ II are always the same, and that massive stars instantaneously explode as SNe II and release metals. This is a natural extension of the work by Yoshii, Tsuiimoto \& Nomoto (1996), in which they selfconsistently modelled the chemical enrichment due to $\mathrm{SNe} \mathrm{Ia}$ and $\mathrm{SNe}$ II.

We have picked out galaxies in dark haloes with $V_{\text {circ }}=220$ $\mathrm{km} \mathrm{s}^{-1}$ and having a similar luminosity to the MW. We have found that when we impose $t_{\mathrm{Ia}}=1.5 \mathrm{Gyr}$, the predictions of our model for such MW-like galaxies agree well with observations of the chemical composition of solar-neighbourhood stars both in the stellar distribution in the $[\mathrm{O} / \mathrm{Fe}]-[\mathrm{Fe} / \mathrm{H}]$ plane and in the frequency distribution of $[\mathrm{Fe} / \mathrm{H}]$. We would like to stress that the other model parameters such as the star formation timescale and SN feedback are the same as in the fiducial model of Nagashima \& Yoshii (2004), in which they found that the model reproduce well many aspects of observed galaxies, such as luminosity functions, cold gas fractions and sizes of galaxies for local galaxies, and the surface brightnesses, velocity dispersions, mass-to-light ratios and metallicities of local dwarf spheroidals. This work also shows that the classical G-dwarf problem van den Bergh 1962; Schmidt 1963; Pagel \& Patchett 1975) is fully resolved in the framework of hierarchical formation of galaxies. Our model passes the new tests, that is, frequency distribution of iron and the abundance pattern of metals, taking into account chemical enrichment due to SNe Ia. As shown in Section 6 the abundance pattern provides an independent constraint on galaxy formation. Therefore this study shows another success for hierarchical galaxy formation.

In our model, disc and bulge stars are treated separately. While the observations of bulge stars still have uncertainties, our model also reproduces well both the distribution of stars in the $[\mathrm{O} / \mathrm{Fe}]-$ $[\mathrm{Fe} / \mathrm{H}]$ plane and the $[\mathrm{Fe} / \mathrm{H}]$ distribution. In particular, although there are only a few bulge stars whose oxygen abundance is observed, our model predicts oxygen-enhanced stars at $[\mathrm{Fe} / \mathrm{H}] \sim 0$, but they are not a dominant fraction. Increasing observational data will provide a strong constraint on bulge formation.

As a by-product, we have obtained the luminosity function of galaxies in Local Group-haloes. Recent high-resolution $N$-body simulations have predicted many more dwarf scale dark haloes than observed satellite galaxies, and this has been considered to be a serious problem (Klypin et al. 1999; Moore et al. 1999). Such an overabundance problem has been solved in the framework of the SA models taking into account effects of reionisation and incompleteness due to significantly low surface brightness (Somerville 2002; Benson et al. 2002b). Our model also shows an ability to solve this problem in a similar manner to those works.

The chemical yields we used are the same as those in Yoshii, Tsuiimoto \& Nomoto (1996), in which an infall model of monolithic collapse was used (Arimoto, Yoshii \& Takahara 1992). 
The similarity between our hierarchical model and the monolithic cloud collapse model suggests that spiral galaxies in a hierarchical universe should have a similar formation history to that modelled by the monolithic cloud collapse model, as shown in Baugh, Cole \& Frenk (1996).

In this paper we have concentrated on investigating the $[\mathrm{O} / \mathrm{Fe}]$ relation and the $[\mathrm{Fe} / \mathrm{H}]$ distribution. Since recent analyses of solarneighbourhood stars have revealed that the age-metallicity relation has a large scatter, it will be useful as a next step to see whether our model succeeds in reproducing this relation as well as the scatter in it (Ibukivama \& Arimoto 2002). Furthermore, while the analysis in this paper focused only on MW-like galaxies, our SA model has the potential ability to investigate other systems simultaneously. To investigate abundance ratios in such systems as the intracluster medium, the stars composing elliptical galaxies, and damped Ly- $\alpha$ systems will provide independent, important clues to understanding galaxy formation. The cosmic explosion rate of $\mathrm{SNe}$ Ia will also give a new insight into both galaxy formation and observational cosmology. We will pursue these topics in future papers.

\section{ACKNOWLEDGMENTS}

We would like to thank Takuji Tsujimoto for useful suggestion and Cedric G. Lacey for reading our paper carefully. We also acknowledge support from the PPARC rolling grant for extragalactic astronomy and cosmology at Durham.

\section{REFERENCES}

Arimoto N., Yoshii Y., 1986, A\&A, 164, 260

Arimoto N., Yoshii Y., 1987, A\&A, 173, 23

Arimoto N., Yoshii Y., Takahara, F., 1992, A\&A, 253, 21

Baugh C. M., Cole S., Frenk C. S., 1996, MNRAS, 283, 1361

Benson A. J., Lacey C. G., Baugh C. M., Cole S., Frenk C. S., 2002a, MNRAS, 333, 156

Benson A. J., Frenk C. S., Lacey C. G., Baugh C. M., Cole S., 2002b, MNRAS, 333, 177

Benson A. J., Frenk C. S., Baugh C. M., Cole S., Lacey C. G., 2003, MNRAS, 343, 679

Berczik P., 1999, A\&A, 348, 371

Binney J., Tremaine S., 1987, Galactic Dynamics, Princeton Univ. Press, Princeton, NJ

Cole S., Aragon-Salamanca A., Frenk C. S., Navarro J. F., Zepf S. E., 1994, MNRAS, 271, 781

Cole S., Lacey C. G., Baugh C. M., Frenk C. S., 2000, MNRAS, 319,168

Edvardsson B, Andersen J., Gustafsson B, Lambert D.L., Nissen

P.E., Tomkin J., 1993, A\&A, 275, 101

Ibukiyama A., Arimoto N., 2002, A\&A, 394, 927

Ishimaru Y., Wanajo S., 1999, ApJL, 511, L33

Kauffmann G., 1996, MNRAS, 281, 475

Kauffmann G., White S. D. M., Guiderdoni B., 1993, MNRAS, 264, 201

Kauffmann G., Charlot S., 1998, MNRAS, 294, 705

Kawata D., 2001, ApJ, 558, 598

Klypin A.A., Kravtsov A.V., Valenzuela O., Prada F., 1999, ApJ, 522,82

Kobayashi C., 2003, MNRAS in press (astro-ph/0310160)

Kobayashi C., Tsujimoto T., Nomoto K., Hachisu I., Kato M., 1998, MNRAS, 503, 155
Kodama T., Arimoto N., 1997, A\&A, 320, 41

Lacey C.G., Fall S.M., 1983, MNRAS, 204, 791

Lacey C.G., Fall S.M., 1985, ApJ, 290154

Lia C., Portinari L., Carraro G., 2002, MNRAS, 330, 821

Maciel W.J., 2001, New Astronomy Reviews, 45, 571

Makino J., Hut P., 1997, ApJ, 481, 83

Martine N.F., Ibata R.A., Bellazzini M., Irwin M.J., Lewis G.F., Dehnen W., 2003, preprint (astro-ph/0311010)

Matteucci F., Greggio L., 1986, MNRAS, 154, 279

Matteucci F., François P., 1989, MNRAS, 239, 885

McWilliam A., Rich R.M., Smecker-Hane T.A., 2003, ApJL, 592, L21

Meléndez J., Barbuy B., 2002, ApJ, 575, 474

Moore B., Ghigna S., Governato F., Lake G., Quinn T., Stadel J., Tozzi P., 1999, ApJL, 524, L19

Nagashima M., Gouda N., Sugiura N., 1999, MNRAS, 305, 449

Nagashima M., Gouda N., 2001, MNRAS, 325, L13

Nagashima M., Totani T., Gouda N., Yoshii Y., 2001, ApJ, 557, 505

Nagashima M., Yoshii Y., Totani T., Gouda N., 2002, ApJ, 578, 675

Nagashima M., Yoshii Y., 2003, MNRAS, 340, 509

Nagashima M., Yoshii Y., 2004, ApJ in press (astro-ph/0404485,

Nakasato N., Nomoto K., 2003, ApJ, 588, 842

Okamoto T., Jenkins A., Eke V.R., Quilis V., Frenk C.S., 2003, MNRAS, 345, 429

Okoshi K., Nagashima M., Gouda N., Yoshioka S., 2004, ApJ, in press

Pagel B.E.J., Patchett B.E., 1975, MNRAS, 172, 13

Pagel B.E.J., Tautvaišienė G., 1995, MNRAS, 276, 505

Ramírez S.V., Stephens A.W., Frogel J.A., DePoy D.L., 2000, ApJ, 120, 833

Raiteri C.M., Villata M., Navarro J.F., 1996, A\&A, 315, 105

Rich R.M., McWilliam A., 2000, Proc. SPIE Vol.4005, p.150

Sadler E.M., Rich R.M., Terndrup D.M., 1996, AJ, 112, 171

Schmidt M., 1963, ApJ, 137, 758

Somerville, R. S. 2002, ApJ, 572, 23

Somerville, R.S., \& Kolatt, T. 1999, MNRAS, 305, 1

Somerville R.S., Primack J. R., 1999, MNRAS, 310, 1087

Somerville R.S., Primack J. R., Faber S.M., 2001, MNRAS, 320, 504

Sugiyama N., 1995, ApJS, 100,281

Sutherland R., Dopita M. A., 1993, ApJS, 88, 253

Thomas D., 1999, MNRAS, 306, 655

Thomas D., Kauffmann G., 1999, in Spectrophotometric dating of stars and galaxies, ed. I. Hubeny, S. Heap \& R. Cornett, Vol. 192 (ASP Conf. Ser.), 261

Tinsley B.M., 1980, Fund. Cosmic. Phys., 5, 287

Tsujimoto T., Nomoto K., Yoshii Y., Hashimoto M., Yanagida Y., Thielemann F.-K., 1995, MNRAS, 277, 945

Tsujimoto T., Shigeyama T., Yoshii Y., 1999, ApJL, 519, L63

van den Bergh S., 1962, ApJ, 67, 486

van den Bergh S., 1992, A\&A, 264, 75

Wyse R.F.G., Gilmore G., 1995, AJ, 110, 2771

Yoshii Y., Tsujimoto T., Nomoto K., 1996, MNRAS, 462, 266

Zoccali M., Renzini A., Ortolani S., Greggio L., Saviane I., Cassisi S., Rejkuba M., Barbuy B., Rich R.M., Bica E., 2003, A\&A, 399, 931

This paper has been typeset from a $\mathrm{T}_{\mathrm{E}} \mathrm{X} / \mathrm{LT}_{\mathrm{E}} \mathrm{X}$ file prepared by the author. 